\title{
Optimizing model of a railroad yard's operations plan based on production scheduling theory
}

\author{
R. Guo ${ }^{1,2}$, J. Guo ${ }^{1} \&$ G. Xie ${ }^{1}$ \\ ${ }^{1}$ School of Information Science and Technology, \\ Southwest Jiaotong University, China \\ ${ }^{2}$ School of Computer and Communication Engineering, \\ Zhengzhou University of Light Industry, China
}

\begin{abstract}
A railroad yard is the basic production unit of rail freight transportation, located at the junction of railway lines, mainly responding to the operations of arriving, sorting, assembling, marshaling, departing; its operation efficiency directly affects the economic benefits of the railroad industry. However, the problem of railroad yards' operations planning not only has a complex model and intractable algorithm, but also a lot of uncertainty factors exist. So searching for an efficient, effective, accurate planning method has always been a difficulty in this field. Different countries, different regions and different production environments vary their description methods, in addition to optimization objectives and constraint conditions. Because of this, it becomes very difficult for researchers from related fields to learn from each other, and the experimental results cannot be compared. So there is an urgent need for building a method of unified modeling based on theory. We consider the fact that operation planning problems of a railroad yard are related to particular production scheduling problems. Then, we use production scheduling theory to describe the rail yard's problem, which can strengthen the theoretical depth and facilitate the research of experts on related fields of scheduling, math, computer and control theory. Combined with the operation feature and overseas and domestic research status, this paper has unified the description method of the operation procedure, variable naming, problem description, objective function and constraint condition. A standard construction method of one switch engine, one hump model was given.
\end{abstract}

Keywords: railroad yards, operations plan, production scheduling, optimal model. 


\section{Introduction}

A railroad yard, also known as a marshalling station, shunting yard or railway yard, is the core of the railway terminal, which is the distribution place for allocating wagon-flows coming from arrival trains to form the new trains. According to statistics, in China, the annual daily number of sorted wagons of a railway network railroad yards is 6000 and the efficiency and quality directly affect the work efficiency and economic benefit of railway transportation [1]. But the planning issue of a railroad yard's operations plan not only has complex model building, involves creating a difficult algorithm for solving it but also has uncertainty factors. Therefore, searching for an efficient real-time planning method is the difficult point in the field. Different countries, station types and operation equipment vary in their description, optimization objectives and constraint conditions making it hard to learn from each other from relevant research results and to compare the experimental results. The development of this field is restricted and there is an urgent need to build up a set of theoretical support for a unified modeling method. The planning issue of a railroad yard's operations plan is a large-scale production scheduling problem, a kind of classical problem of combination and optimization. From the point of view of computational complexity, this kind of problem has NP properties and it is hard to find the optimal solution. The approximate solution is usually obtained by using the approximate algorithm or the heuristic algorithm.

In this paper, combined with the modeling method of production scheduling theory, we build a wagon-flow allocation model of single directional, single pushing and single humping. This model is established on the optimal goal for the shortest dwell time of all cars during a stage plan time and with some constraint conditions such as car flows joining relation, destinations, full axis, rail cars in arrival or departure trains, hump operation type, sorting sequence, assembling sequence etc.

\section{Problem statement}

\subsection{Production scheduling problem}

The production scheduling problem [2] is one of the representative problems of combinatorial optimal problems in operations theory. It means making the reasonable production decision under the known condition of production tasks. According to the expended goals, it should determine how to most efficiently allocate the limited human and material resources to different tasks in time order. If one only considers the processing sequence of the tasks, it is a sequencing problem in mathematics, which is an urgent problem to be solved in the process of manufacturing production management [3]. In 1954, after the first article which described a permutation flow shop scheduling problem of two machines was published [4], in the following 60 years, scheduling problems have been the concern of researchers in the fields of mathematics, computers, industrial engineering and economic management. 
According to the literature [5-9], the research can be divided into the following 5 stages: for the first stage (1950s-1960s), its technical characteristics are a combination of analysis; for the second stage (1960s-1970s), the main technical features are branch and bound, and dynamic programming; for the third stage (1970s-1980s), the main technical feature is computational complexity theory; for the fourth stage (1980s-1990s), the main technical feature is the approximation algorithm combined with heuristic strategies; for the fifth stage (1990s to date), the main technical features are theories of real time and uncertainty modern scheduling. The research of production scheduling has been accompanied by the development of mathematics and computer science. For different production processes, the mathematical models of the different production scheduling problems vary. As an easy description, in 1967, Conway et al. [10] proposed a method of four parameters representing a scheduling problem. Based on that, in 1979, Graham et al. [11] proposed a three-parameter representation method. Then a large number of studies followed using the description of three parameters $\alpha|\beta| \gamma$, and the definitions of Pinedo in [12] can be associated with our question as follows.

\subsubsection{The possible machine environments specified in the $\alpha$ field} Identical machines in parallel $(P m)$ : There are $m$ identical machines in parallel.

Flow shop $(F m)$ : There are $m$ machines in series. Each job has to be processed on each one of the $m$ machines. All jobs have to follow the same route, i.e., they have to be processed first on machine 1 , then on machine 2 , and so on.

Flexible flow shop $\left(F F_{c}\right)$ : A flexible flow shop is a generalization of the flow shop and the parallel machine environments. Instead of $m$ machines in series there are $c$ stages in series with at each stage a number of identical machines in parallel. Each job has to be processed first at stage 1, then at stage 2, and so on. A stage functions as a bank of parallel machines; at each stage job $j$ requires processing on only one machine and any machine can do. The queues between the various stages may or may not operate according to the First Come First Served (FCFS) discipline. (Flexible flow shops have in the literature at times also been referred to as hybrid flow shops and as multiprocessor flow shops.)

\subsubsection{The processing restrictions and constraints specified in the $\beta$ field}

Release dates $\left(r_{j}\right)$ : If this symbol appears in the $\beta$ field, then job $j$ cannot start its processing before its release date $r_{j}$. If $r_{j}$ does not appear in the $\beta$ field, the processing of job $j$ may start at any time.

Preemptions ( prmp): Preemptions imply that it is not necessary to keep a job on a machine, once started, until its completion. When preemptions are allowed prmp is included in the $\beta$ field; when prmp is not included, preemptions are not allowed.

Permutation (prmu): A constraint that may appear in the flow shop environment is that the queues in front of each machine operate according to the First In First Out (FIFO) discipline. 
Blocking (block): Blocking is a phenomenon that may occur in flow shops. If a flow shop has a limited buffer in between two successive machines, then it may happen that when the buffer is full the upstream machine is not allowed to release a completed job.

\subsubsection{Possible objective functions to be minimized in the $\gamma$ field}

Total weighted completion time $\left(\sum w_{j} C_{j}\right)$ : The sum of the weighted completion times of the $n$ jobs gives an indication of the total holding or inventory costs incurred by the schedule. The sum of the completion times is in the literature often referred to as the flow time. The total weighted completion time is then referred to as the weighted flow time.

\subsection{Technical operations of railroad yards}

Each railroad yards needs to set up a number of yards according to its own transport production demand; it can complete multi-destination technical operation such as train arrival and departure operations, car sorting and assembly operations. The function partition and flexible use of each yard are determined by the rational layout of the station, the capacity of the technical equipment, and the cars meeting a balance between inbound and outbound trains. Based on the practical operational process, the yards can be divided into functions of a receiving yard, a classification yard and a departure yard. In addition, there can be a hump in the linking area between the receiving yard and the classification yard, and also the pulling out tracks in the linking area between the classification yard and the departure yard. The hump has a certain degree of slope, so that it can help the wagon flow under potential energy to slip into the classification yard. Therefore it is always used for sorting. The pulling out tracks are the flat tracks used for marshalling or picking up, and also for no hump car sorting. Figure 1 gives a station chart of a bidirectional three-grade six-yard transversal type railroad yard which is commonly used for larger operations.

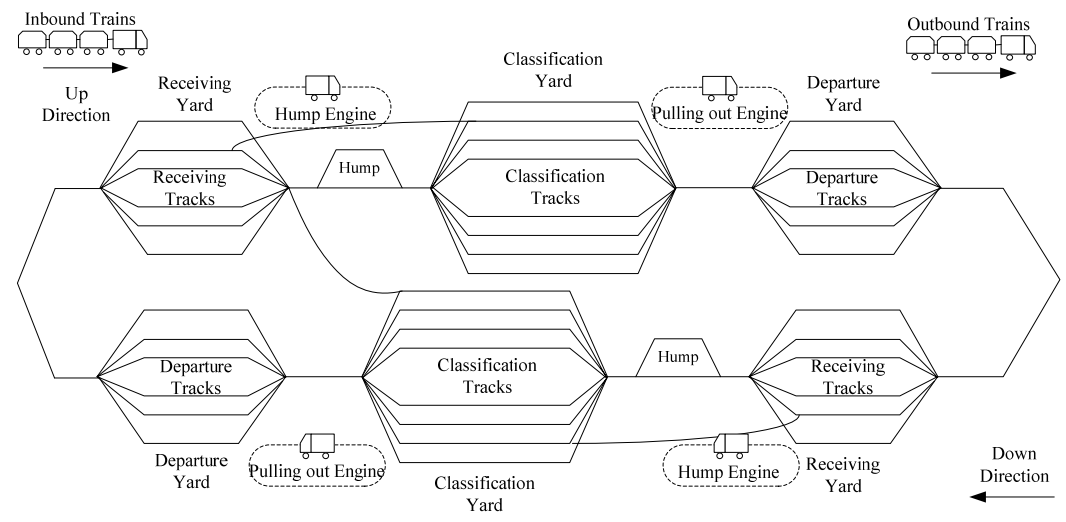

Figure 1: Station chart of a bidirectional three-grade six-yard transversal type railroad yard. 
Railroad yards have perfect transportation equipment in that they can carry out all of the work flow in the station such as arriving, sorting, accumulating, marshalling and departing.

There are many kinds of definitions about the functional assignment in the classification yard and receiving yard (see [13-17]). In this paper, we review one of the situations: the arriving technical operation refers to the task of meeting the arrival train on the receiving track and of its being inspected by an inspector group etc.; the sorting technical operation refers to disassembling the inbound train flow or train set onto the special track of the classification yard via the hump or pull out track according to the cars' destinations; the accumulating technical operation refers to the car halting process which starts from the time of humping into the classification yard and ends at the time of pulling out to the departure yard; the marshalling technical operation refers to the shunting operation which needs to select accumulated cars by the pulling out engine to make up wagon flow according to the rules of the freight train formation plan, the train diagram, and the regulations governing the railway technical operation on the pulling out tracks; the departure technical operation refers to the setup operation for the departing train in the departure yard.

\subsection{The relationship between production scheduling and railway yard operation scheduling}

The railway yard operations problem is the need to organize a variety of productive resources to complete the work of meeting arriving trains and making sure trains depart day and night according to production demand; it is a large-scale complex production scheduling problem. In China, the schedulers organize the production scheduling plan in accordance with a daily shift working plan which is made firstly by railway administration. According to the specific circumstances of the current station, taking 3-4 hours as a stage, they adjust the deviation in the daily shift plan and gradually make detailed operation plans to achieve the production indexes. The operation scheduling can be divided into the daily shift working plan, the stage plan and the shunting operation plan. The relationships between the different levels of the plans are shown in Figure 2.

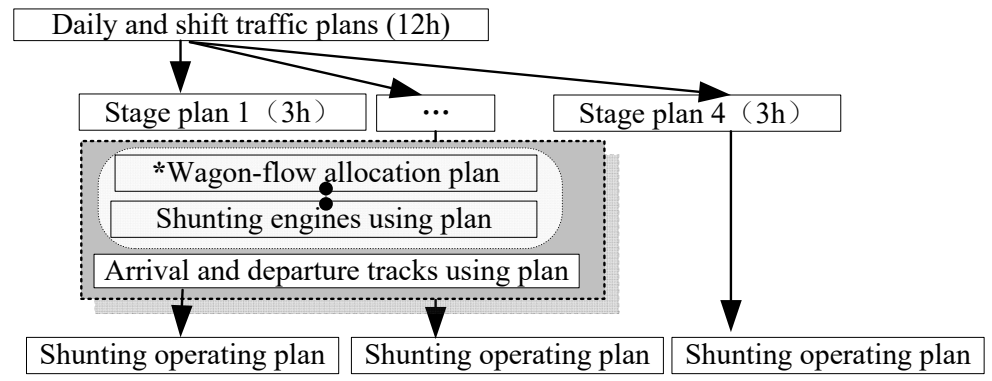

Figure 2: The different levels of the railroad yard scheduling operation plans. 
The stage plan is the core of the whole plan, which is like a connecting link between the up level plan and the down level plan. Establishment of a stage plan mainly includes car assignment, the humping sequence, the marshalling sequence, hump engine scheduling, pulling out engine scheduling and arrival and departure track occupation, through setting a wagon-flow allocation plan, a shunting engine usage plan and an arrival and departure track usage plan. The focus of the stage plan is the wagon-flow allocation plan which forms the basis for the other two plans. The wagon-flow allocation plan not only needs to consider the requirements of the departure trains in the current stage, but also the factors of humps, engines, and tracks comprehensively. It mainly determines the departure train: cars coming from which arrival trains, the detailed destinations, and the number of cars in one destination. The destination and all car numbers must obey the regulations of the formation plan. It is not only the embodiment of coordinated operation in sorting and assembling, but also an optimal problem of reasonable selection and collocation. The process of wagon-flow allocation can be divided into dynamic wagon-flow allocation and static wagon-flow allocation [18]. Dynamic wagonflow allocation mainly solves how to set the order of sorting based on the demand of the outbound trains. Static wagon-flow allocation studies focus on how to make train allocation under the condition of determined sorting order. Therefore, the key to finding the optimum wagon-flow allocation plan is to choose a rational sorting order. The wagon-flow allocation is discussed in the following paragraphs.

The research into the railway yard operation problem involves many technical terms, which makes it difficult to understand for experts in the field of other scheduling problems from other majors. Therefore, we hope that this problem can be changed from a specific scheduling problem of railway yards to a more general production scheduling model. The forms of the production scheduling problem are various, and it is difficult to unify. In fact, many technical terms originate from manufacturing scheduling problems which first attracted attention, such as machine, job, operation and order. In order to unify the terms, the specific scheduling problems can be abstracted and converted into a standard form of three parameters.

Based on the description of the deterministic production scheduling problem, the model distributes receiving tracks, inspectors, humping engine, hump, classification tracks, pulling out engine and the number of departure tracks to different buffers, and makes an inbound train correspond to a job and technical operations correspond to different machines. So, the description of a wagon-flow allocation problem in a specific railway yard is as follows.

The total number of inbound trains is $n$, where the trains have different priorities. The yards consist of 18 receiving tracks, 2 humping engines, 1 piece of hump equipment, 30 classification tracks, 1 pulling out engine, 14 departure tracks, and 2 departure technical operation groups. Each inbound train needs four operating procedures in order: they are arriving technical operation, sorting technical operation, marshalling technical operation and departing technical operation. Each procedure can only be processed on one machine with no preempt. When the buffer is without enough spare space, the upstream machine can't release the job. Each procedure needn't meet the first in first out rule, that is to say the 
model has no permutation constraint. In addition, the wagon-flow allocation plan should be constrained by the requirements of the formation plan and the train diagram; this problem also belongs to the scheduling problem and has a due date. As the stage plan is only a part of the daily shift working plan and the jobs have different arrival times, the total completion time is not suitable to this problem as it can't recognise the efficiency of inbound trains. We adopt the total wagon-flow dwell time $\left(C_{j}{ }^{\prime}\right)$ on the station during a stage plan time as the optimal function. Based on the above analysis, the problem can be described as a flexible flow shop scheduling problem with blocking. The problem described by the three parameters is: $F F_{4} \mid r_{j}$, block $\mid \sum w_{j} C_{j}{ }^{\prime}$. Figure 3 illustrates the job flow of the problem.

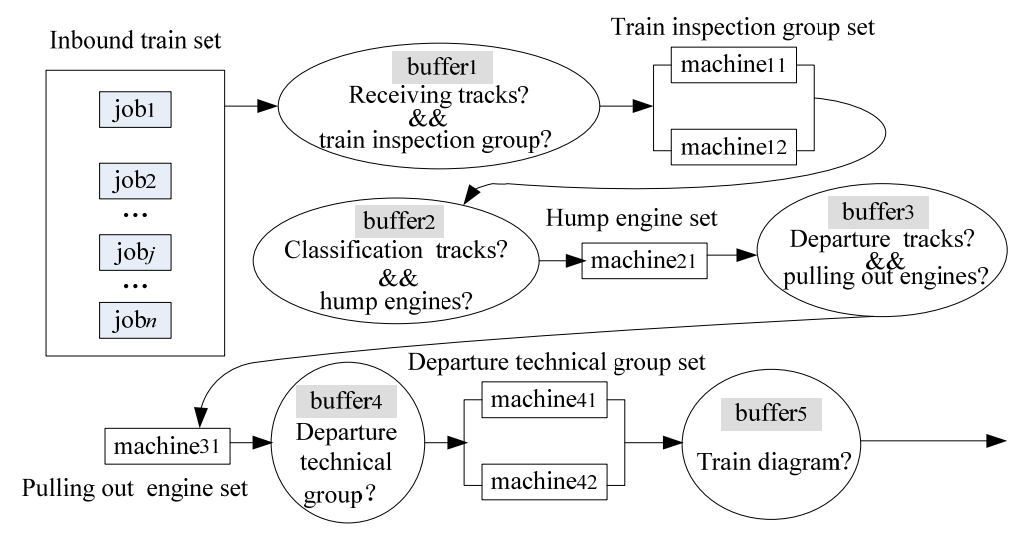

Figure 3: The job flow of the wagon-flow allocation problem.

\section{The optimal model of the scheduling problem of railroad yards}

The model of single pushing and single humping is a fundamental scheduling formation in a wagon-flow allocation problem. Based on this model, many problems of other models can be transformed or expanded. Other modeling problems refer to double pushing and double humping, the yards that receive exchange cars, empty car distribution and multi-objective optimization. Compared with others, sorting is the core operation. We assume that under the condition of sufficient operation resources, which include the group of inspectors, the tracks of all kinds of yards, and the marshalling sequence being equal to the departure sequence according to the train diagram, the problem is simplified to a sorting sequence optimal problem. When we assume the case of one humping engine and one pulling out engine, the problem can be described as $F_{2} \mid r_{j}$, block $\mid \sum w_{j} C_{j}{ }^{\prime}$ in the format of production scheduling theory.

For the characteristics of the wagon-flow problem, the conventions are given as follows: the total number of trains including which are halting in the classification yard, having arrived or being expected to arrive in the receiving yard is $n+1$, we assume that all of the cars are in the classification yard at the beginning 
of the stage plan at the $0^{\text {th }}$ inbound train; the total number of outbound trains in the time of a stage plan is $m+1$, where we assume that the $(m+1)^{\text {th }}$ outbound train refers to all of the cars not assembled to any outbound train at the end of the stage plan; the maximum number of sorting intervals is defined as $\mathrm{K}$, where sorting interval refers to the time window between the beginning and the ending of a whole sorting operating process, assuming $T_{0}$ to be the starting time of a stage plan, and letting the stage plan operating time be 3-4 hours; outbound trains must obey the train diagram to operate, but the outbound train is allowed to depart whilst not meeting the constraint of full axis so as not to waste the operation curves of the train diagram. The definition of the symbols used in the model are shown in Table 1.

Table 1: Symbol definition.

\begin{tabular}{|c|c|c|}
\hline Symbol & Definition & Vague range \\
\hline $\mathrm{D}$ & inbound train & symbolic constant \\
\hline $\mathrm{C}$ & outbound train & symbolic constant \\
\hline $\mathrm{CF}$ & departure technical operation & symbolic constant \\
\hline $\mathrm{BZ}$ & marshalling technical operation & symbolic constant \\
\hline JT & sorting technical operation & symbolic constant \\
\hline DD & receiving technical operation & symbolic constant \\
\hline JTQJ & sorting interval & symbolic constant \\
\hline BZQJ & marshalling interval & symbolic constant \\
\hline$j$ & inbound train numbering & $j=0,1, \cdots, n$ \\
\hline$i$ & outbound train numbering & $i=1, \cdots, m, m+1$ \\
\hline$f$ & destination & $f={ }^{\prime} 1,{ }^{\prime}, 2, \cdots,{ }^{\prime} N$ ' \\
\hline$k$ & sorting interval numbering & $k=1,2, \cdots, K$ \\
\hline$h$ & marshalling interval numbering & $h=1,2, \cdots, m$ \\
\hline$F$ & the set of all destinations on the railroad yards & $F=\left\{{ }^{\prime} 1, \cdots, \cdots, f, \cdots,{ }^{\prime} N^{\prime}\right\}$ \\
\hline$D_{j}$ & the $j^{\text {th }}$ inbound train & $D_{j}=D_{0}, D_{1}, \cdots, D_{n}$ \\
\hline$C_{i}$ & the $i^{\text {th }}$ outbound train & $C_{i}=C_{1}, C_{2}, \cdots, C_{m+1}$ \\
\hline$w_{j}$ & the weight of $\mathrm{D}_{j}^{\prime}$ priority & \\
\hline$F_{\mathrm{D}, j}$ & the set including all of $f \mathrm{~s}$ in $\mathrm{D}_{j}$ & $F_{\mathrm{D}, j} \subseteq F$ \\
\hline$F_{\mathrm{C}, i}$ & $\begin{array}{l}\text { the set including all of } f \mathrm{~s} \text { conforming to } C_{i} \text { 's } \\
\text { marshalling demand }\end{array}$ & $F_{\mathrm{C}, i} \subseteq F$ \\
\hline$D_{j, f}$ & the set of cars in $D_{j}$ on the destination of $f$ & $D_{j, f} \subseteq D_{j}$ \\
\hline$|X|$ & the number of set $X$ & nonnegative integer \\
\hline$C_{i, j}^{f}$ & $\begin{array}{l}\text { the set of wagons coming from } D_{j, f} \text { and } \\
\text { allocated to } C_{i} \text { on the destination of } f\end{array}$ & $C_{i, j}^{f} \subseteq D_{j, f}$ \\
\hline$x_{i, j}^{f}$ & the number of $C_{i, j}^{f}$ & nonnegative integer \\
\hline$T_{\mathrm{C}, i}$ & departure time of $C_{i}$ & nonnegative integer \\
\hline$T_{\mathrm{D}, j}$ & arrival time of $D_{j}$ & nonnegative integer \\
\hline$t_{\mathrm{CF}, i}$ & departure operating time of $C_{i}$ & nonnegative integer \\
\hline$t_{\mathrm{BZ}, i}$ & marshalling operating time of $C_{i}$ & nonnegative integer \\
\hline$t_{\mathrm{JT}, j}$ & sorting operating time of $D_{j}$ & nonnegative integer \\
\hline$t_{\mathrm{DD}, j}$ & arriving operating time of $D_{j}$ & nonnegative integer \\
\hline & the number of $C_{i}$ 's full axis & positive integer \\
\hline$D_{\mathrm{JTQJ}, k}$ & $D_{j}$ assigned to the $k^{\text {th }}$ soring interval & $D_{\mathrm{JTQJ}, k} \in\left\{D_{1}, \cdots, D_{n}\right\}$ \\
\hline$T_{\mathrm{JTKS}, k}$ & the start time of the $\mathrm{k}^{\text {th }}$ soring interval & nonnegative integer \\
\hline$C_{\mathrm{BZQJ}, h}$ & $C_{i}$ assigned to the $h^{\text {th }}$ marshalling interval & $C_{\mathrm{BZQJ}, h} \in\left\{C_{1}, C_{2}, \cdots, C_{m}\right\}$ \\
\hline$T_{\mathrm{BZKS}, h}$ & the start time of the $h^{\text {th }}$ marshalling interval & nonnegative integer \\
\hline
\end{tabular}


Table 1: $\quad$ Continued.

\begin{tabular}{|c|c|c|}
\hline Symbol & Definition & Vague range \\
\hline$Y_{i, j}^{T}$ & $\begin{array}{l}0-1 \text { variables represent the cars matching } \\
\text { relation between } D_{j} \text { and } C_{i} \text { according time }\end{array}$ & $Y^{T}{ }_{i, j}=0,1$ \\
\hline$Y_{i, j}^{f}$ & $\begin{array}{l}0-1 \text { variables represent whether the direction of } \\
f \text { conforms to } C_{i}^{\prime} \text { formulation rules according } \\
\text { to the marshalling plan }\end{array}$ & $Y_{i, j}^{f}=0,1$ \\
\hline$Y_{\text {JTQJ }, k}$ & $\begin{array}{l}0-1 \text { variables represent whether } D_{j} \text { can be } \\
\text { disassembled on the } k^{\text {th }} \text { soring interval }\end{array}$ & $Y_{\mathrm{JTQJ}, k}^{j}=0,1$ \\
\hline$Y_{\text {BZQJ }, h}^{i}$ & $\begin{array}{l}0-1 \text { variables represent whether } C_{i} \text { can be } \\
\text { assembled on the } h^{\text {th }} \text { marshalling interval }\end{array}$ & $Y_{\mathrm{BZQJ}, h}^{i}=0,1$ \\
\hline
\end{tabular}

The production environment, the processing restrictions and constraints, and the optimal functions of the model can be described as follows.

\subsection{The production environment}

1 humping engine and 1 pulling out engine, so it belongs to a 2 machine flow shop problem.

\subsection{The processing restrictions and constraints}

(1) Wagon-flow joining relation constraints:

$$
\begin{aligned}
& Y^{T} T_{i j}=\left\{\begin{array}{ll}
0 & T_{\mathrm{C}, i}-t_{\mathrm{CF}, i}-t_{B Z, i}-t_{\mathrm{JT}, j}-t_{D D, j}-T_{\mathrm{D}, j}<0 \\
1 & T_{\mathrm{C}, i}-t_{\mathrm{CF}, i}-t_{B Z, i}-t_{\mathrm{JT}, j}-t_{D D, j}-T_{\mathrm{D}, j} \geq 0
\end{array}, \quad 1 \leq i \leq m, 0 \leq j \leq n ;\right. \\
& T_{\mathrm{C}, i}, t_{\mathrm{CF}, i}, t_{\mathrm{BZ}, i}, t_{\mathrm{JT}, j}, t_{\mathrm{DD}, j}, T_{\mathrm{D}, j} \geq 0, \quad 1 \leq i \leq m, 0 \leq j \leq n ; \\
& x_{i, j}^{f} \leq Y_{i, j}^{T_{i}} * \mid D_{j, f}, 1 \leq i \leq m, 0 \leq j \leq n, 1 \leq f \leq N \text {; }
\end{aligned}
$$

Equation (1) is considered for the wagon-flow joining relation between $D_{j}$ and $C_{i}$. If it is satisfied, $Y^{T}{ }_{i, j}=1$; otherwise $Y^{T}{ }_{i, j}=0$. Equation (2) shows that all the values of the time variables can't be negative. Equation (3) means the cars of $D_{j}$ can be assembled to $C_{i}$ if and only if $Y_{i, j}^{T}=1$.

(2) Destination constraints:

$$
\begin{aligned}
& Y_{i, j}^{f}=\left\{\begin{array}{cc}
0 & \left(\left(f \in F-F_{\mathrm{D}, j} \cap F_{\mathrm{C}, i}\right) \wedge\left(F_{\mathrm{D}, j} \cap F_{\mathrm{C}, i} \neq \varnothing\right) \vee\left(F_{\mathrm{D}, j} \cap F_{\mathrm{C}, i}=\varnothing\right)\right) \\
1 & \left(\left(f \in F_{\mathrm{D}, j} \cap F_{\mathrm{C}, i}\right) \wedge\left(F_{\mathrm{D}, j} \cap F_{\mathrm{C}, i} \neq \varnothing\right)\right)
\end{array},\right. \\
& 1 \leq i \leq m, 0 \leq j \leq n, 1 \leq f \leq N \text {; } \\
& x_{i, j}^{f} \leq Y_{i, j}^{f} *\left|D_{j, f}\right|, 1 \leq i \leq m, 0 \leq j \leq n, 1 \leq f \leq N \text {; }
\end{aligned}
$$

Equation (4) shows that $Y_{i, j}^{f}=1$ if and only if the destination $f$ of $D_{j}$ s cars included in the $C_{i}$ destination set; otherwise $Y_{i, j}^{f}=0$. Equation (5) means that the cars of $D_{j}$ can be assembled to $C_{i}$ if and only if $Y_{i, j}^{f}=1$.

(3) The car number assembling to the outbound train constraint:

$$
\sum_{j=0}^{n} \sum_{f=1}^{d} x_{i, j}^{f} \leq r_{i}, 1 \leq i \leq m
$$

(4) The car number constraints between the inbound train and outbound train:

$$
\sum_{i=1}^{m} \sum_{f \in F_{\mathrm{D}, j}} x_{i, j}^{f} \leq \sum_{f \in F_{\mathrm{D}, j}}\left|D_{j, f}\right|, 0 \leq j \leq n
$$




$$
\sum_{j=0}^{n} \sum_{f \in F_{\mathrm{C}, i}} x^{f}{ }_{i, j} \leq \sum_{j=0}^{n} \sum_{f \in F_{\mathrm{C}, i}}\left|D_{j, f}\right|, 1 \leq i \leq m ;
$$

Equation. (7) shows that the number of cars in $D_{j}$ must be greater than or equal to all the number of cars in $C_{i}$ which comes from $D_{j}$; Equation (8) means that the number of cars in $C_{i}$ must be less than or equal to the number of cars in $D_{j}$ which satisfies the destination requirement of $C_{i}$.

(5) Hump and humping engine constraints (single pushing and single humping): if $D_{j}$ can be arranged to sort on the $k^{\text {th }}$ sorting interval, it must meet the following constraints:

$$
\begin{aligned}
& Y_{\mathrm{JTQJ}, k}=\left\{\begin{array}{ll}
0 & D_{\mathrm{JTQJ}, k} \neq D_{j} \\
1 & D_{\mathrm{JTQJ}, k}=D_{j}
\end{array}, 1 \leq j \leq n, 1 \leq k \leq K ;\right. \\
& \sum_{j=1}^{n} Y^{j}{ }_{\mathrm{JTQJ}, k}=1,1 \leq k \leq K \text {; } \\
& \sum_{k=1}^{K} Y^{j}{ }_{\mathrm{JTQJ}, k}=1,1 \leq j \leq n \text {; } \\
& T_{\mathrm{JTKS}, k}+\sum_{j=1}^{n} Y_{\mathrm{JTQJ}, k}^{j} * t_{\mathrm{JT}, j} \leq T_{\mathrm{JTKS}, k+1}, 1 \leq k \leq K-1 ; \\
& \left\{\begin{array}{c}
T_{\mathrm{JTKS}, 1} \geq \max \left(T_{0}, \sum_{j=1}^{n} Y^{j}{ }_{\mathrm{JTQJ}, 1} *\left(T_{\mathrm{D}, j}+t_{\mathrm{DD}, j}\right)\right), k=1 \\
T_{\mathrm{JTKS}, k} \geq \max \left(\begin{array}{l}
T_{\mathrm{JTKS}, k-1}+\sum_{j=1}^{n} Y^{j}{ }_{\mathrm{JTQJ}, k-1} * t_{\mathrm{JT}, j}, \\
\sum_{j=1}^{n} Y^{j}{ }_{\mathrm{JTQJ}, k} *\left(T_{\mathrm{D}, j}+t_{\mathrm{DD}, j}\right)
\end{array}\right), 1<k \leq K
\end{array} ;\right. \\
& \sum_{j=1}^{n} \sum_{k=1}^{K} Y^{j}{ }_{\mathrm{JTQJ}, k} * t_{\mathrm{JT}, j} \leq t \\
& x_{i, j}^{f}=Y_{\mathrm{JTQ}, k} x_{i, j}^{f}, 1 \leq j \leq n, 1 \leq i \leq m ;
\end{aligned}
$$

Equation (9) shows that if $D_{j}$ has been arranged to sort on the $k^{\text {th }}$ sorting interval, then the value of $Y_{\mathrm{JTQJ}, k}^{j}$ is equal to 1 ; otherwise the value of $Y_{\mathrm{JTQJ}, k}$ is equal to 0 . Equation (10) shows that one sorting interval only can arrange one inbound train operation. Equation (11) shows that one inbound train only can occupy one sorting interval. Equation (12) shows that only when the upstream inbound train has completed its sorting operation can the current inbound train begin its operation by the same humping engine. Equation (13) means that only when $D_{j}$ 's earliest sorting time is less than or equal to $T_{\mathrm{JTKS}, k}$ can $D_{j}$ be arranged to the $k^{\text {th }}$ sorting interval. Equation (14) means that the total inbound train sorting operating time must be less than or equal to the stage plan time $T$. Equation (15) shows that the number of cars coming from the unassembled trains can't be the same as the number of $x_{i, j}^{f}$.

(6) Pulling out engine constraints:

$$
Y_{\mathrm{BZQJ}, h}^{i}=\left\{\begin{array}{ll}
0 & C_{\mathrm{BZQJ}, h} \neq C_{i} \\
1 & C_{\mathrm{BZQJ}, h}=C_{i}
\end{array} \quad 1 \leq h, i \leq m ;\right.
$$




$$
\begin{gathered}
\sum_{i=1}^{m} Y_{\mathrm{BZQJ}, h}^{i}=1,1 \leq h \leq m ; \\
\sum_{h=1}^{m} Y^{i}{ }_{\mathrm{BZQJ}, h}=1,1 \leq i \leq m ; \\
T_{\mathrm{BZKS}, h}+t_{\mathrm{BZ}, i} \leq T_{\mathrm{BZKS}, h+1}, 1 \leq i \leq m, 1 \leq h \leq m-1 ; \\
T_{\mathrm{BZKS}, h} \leq T_{\mathrm{C}, i} t_{\mathrm{CF}, i}-t_{\mathrm{BZ}, i}, 1 \leq h, i \leq m ; \\
\sum_{i=1}^{m} \sum_{h=1}^{m} Y_{\mathrm{BZQJ}, h} * t_{\mathrm{BZ}, i} \leq t ; \\
x_{i, j}^{f}=Y^{i}{ }_{\mathrm{BZQJ}, h}{ }^{*} x_{i, j}^{f}, 1 \leq j \leq n, 1 \leq i \leq m ;
\end{gathered}
$$

Equation (16) shows that if $C_{i}$ has been arranged to assemble on the $h^{\text {th }}$ marshalling interval, then the value of $Y_{B Z Q J, h}$ is equal to 1 ; otherwise the value of $Y_{B Z Q J, h}$ is equal to 0 . Equation (17) shows that one marshalling interval only can arrange one outbound train operation. Equation (18) shows that one outbound train only can occupy one marshalling interval. Equation (19) shows that only when the upstream outbound train has completed its marshalling operation can the current outbound train begin its operation by the same pulling out engine. Equation (20) means that only when $C_{i}$ 's latest marshalling time is greater than or equal to $T_{\mathrm{BZKS}, h}$ can $C_{i}$ be arranged to the $h^{\text {th }}$ marshalling interval. Equation (21) means that the total outbound train marshalling operating time must be less than or equal to the stage plan time $T$. Equation (22) shows that if $C_{i}$ hasn't accessed any marshalling interval, then the number of cars in the classification yard can't be the same as the number of $x_{i, j}^{f}$.

\subsection{The optimal functions}

Let $x_{i, j}^{f}$ be the decision variable, making the weighted total wagon-flow dwell time on the station during the stage plan time be the optimal function, as follows:

$$
\min z\left(x_{i, j}^{f}\right)=\sum_{i=1}^{m+1} \sum_{j=0}^{n} \sum_{f \in F_{\mathrm{C}, i} \cap F_{\mathrm{D}, j}} w_{j} x_{i, j}^{f} *\left(T_{\mathrm{C}, i}-T_{\mathrm{D}, j}\right) .
$$

\section{Conclusions}

The railroad yard scheduling problem is more complicated than the same type of production scheduling problem. In any scheduling table, there is another NP problem which refers to the resource matching problem. Combined with different problems, using the historical data to generate the heuristic rules, the key point for further research is to build up a concise model and find an efficient algorithm.

\section{Acknowledgements}

This research has been supported in part by major research projects of the China Railway Corporation (2014X008-A), key projects of the research project of the China Railway Corporation (2015X007-J) and the Educational Committee of Henan Province of China (15A520004). 


\section{References}

[1] Jianye Song, Jinbao Xie. Basis on organization of train operation. Beijing: China Railway Publishing House, 2005.

[2] Wanliang Wang, Qidi Wu. Intelligent algorithm and application for production scheduling. Beijing: Science Press, 2007.

[3] Guochun Tang. Review of basic concepts of scheduling theory. Journal of Chongqing Normal University, 2012, 29(4): 1-11.

[4] Johnson S. M. Optimal two- and three-stage production schedules with setup times included, Naval Research Logistics Quarterly, 1954, 1(1): 61-68.

[5] Stephen C. Graves. A review of production scheduling. Operations Research, 1981, 29(4): 646-675.

[6] A.S. Jain, S. Meeran. Deterministic job-shop scheduling: past, present and future. European Journal of Operational Research, 1999, 133: 390-434.

[7] Jatinder N.D. Gupta, Edward F. Stafford Jr. Flowshop scheduling research after five decades. European Journal of Operational Research, 2006, 169: 699-711.

[8] Chris N. Potts, Vitaly A. Strusevich. Fifty years of scheduling: a survey of milestones. Journal of the Operational Research Society, 2009, 60: 41-68.

[9] Beunhard Korte, Jens Vygen. Combinatorial optimization: theory and algorithms (3rd ed.). Springer-Verlag, Berlin, Heidelberg, 2006.

[10] Conway, R., Maxwell, W., Miller, L. Theory of Scheduling, AddisonWesley, Reading, MA, 1967.

[11] Graham, R., Lawler, E., Lenstra, et al. Optimization and approximation in deterministic sequencing and scheduling: A survey. Annals of Discrete Mathematics, 1979, 5: 287-326.

[12] Pinedo, M. Scheduling: Theory, Algorithms, and Systems (3rd ed.). New York: Prentice-Hall, 2008.

[13] Shi Tie, Xuesong Zhou. A mixed integer programming model for optimizing multi-level operations process in railroad yards. Transportation Research Part B, 2015, 80: 19-30.

[14] Hai Wang, Jiangang Jin, Maokai Lin. Solving the railway yard operation problem: greedy heuristics, integer programming models, and waiting time approximations. Cambridge, MA: MIT Press, 2013: 1-14.

[15] Florian Jaehn, Johannes Rieder, Andreas Wiehl. Minimizing delays in a shunting yard, Springer-Verlag, Berlin, Heidelberg, OR Spectrum, 2015, 37 : 407-429.

[16] Nils Boysen, Malte Fliedner, Florian Jaehn. Shunting yard operations: Theoretical aspects and applications. European Journal of Operational Research, 2012, 220:1-14.

[17] Shiwei He, Rui Song, Sohail S. Chaudhry. An integrated dispatching model for rail yards operations. Computers \& Operations Research 2003, 30: 939 966.

[18] Ciguang Wang. Research on the model and algorithm of dynamic wagon flow allocating in a marshalling station. Journal of The China Railway Society, 2004, 26(1): 1-6. 